\title{
Plasmid-mediated quinolones resistance in clinically important bacteria
}

\author{
M. Omidvar Panah ${ }^{1}$, M. Najafi ${ }^{1}, \underline{\text { A. Peymani }}^{1}$
}

\author{
${ }^{1}$ Medical Microbiology Research Center, Qazvin University of Medical Sciences, Qazvin, Iran \\ Corresponding Address: Amir Peymani, Medical Microbiology Research Center, Velayat Hospital, Qazvin University of \\ Medical Sciences, Qazvin, Iran \\ Tel: +98-28-33324971, Email: a.peymani@gmail.com \\ Received: 7 Apr 2018; Accepted: 22 May 2018
}

\section{Abstract}

Quinolones are synthetic and commonly used antibiotics for treatment of multiple clinical infections in the world. Quinolones are clinically important antibiotics, as an ideal component, because of high potency, broad-spectrum activity, good bioavailability and a potentially low incidence of side-effects. These antibiotics are not originated from biological source. In addition to chromosomal mutations in the target genes which confer resistance to these antibiotics, in recent years, plasmids-mediated quinolone resistance (PMQR) have made difficult to treat infections caused by resistant organisms. PMQR plays a very important role in resistance to these antibiotics due to the rapid spread between the bacteria. So far, three types of PMQR have been identified, including the aac(6')-Ib-cr, the qepA efflux pump and the qnr proteins. In this research, the role of qnr proteins in the development of drug resistance to the quinolone compounds has been studied.

Keywords: Quinolone, Drug resistance, Qnr protein

Citation: Omidvar Panah M, Najafi M, Peymani A. Plasmid-mediated quinolones resistance in clinically important bacteria. J Qazvin Univ Med Sci 2018; 22(2): 90-99. 


\title{
مقاومت به كينولونها به واسطه يالاسميد در باكترىهاى با اهميت بالينى
}

\author{
مريم اميدوار يناه'، مريم نجفى'، دكتر امير بيمانى' \\ "مركز تحقيقات ميكروبشناسى يزشكى دانشخاه علوم يزشكى قزوين، قزين، ايران
}

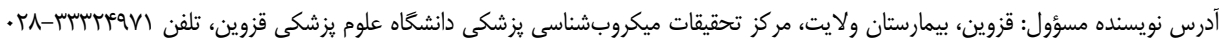

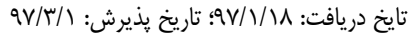

داروهاى كينولون مواد ضدميكروبى صناعى و از آنتى بيوتيكهاى رايج مصرفى در دنيا هستند كه در درمان عفونتهاى متعدد بالينى مـورد استفادهاه

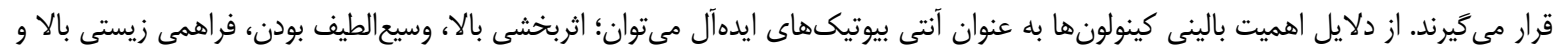

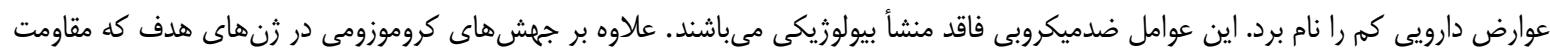

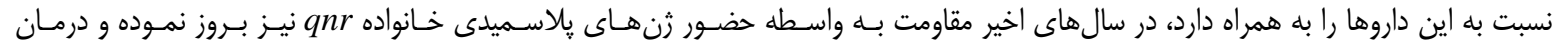

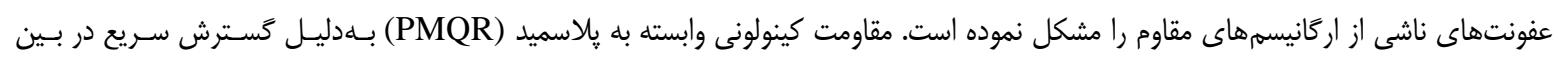

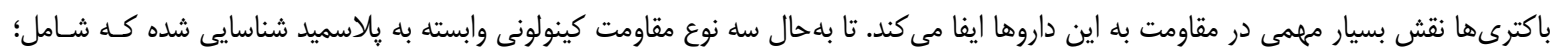

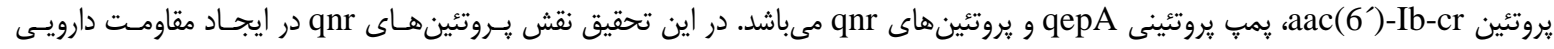

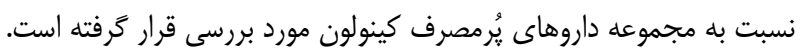

كليدوازهها: كينولون، مقاومت دارويى، يروتئين qnr

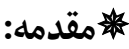

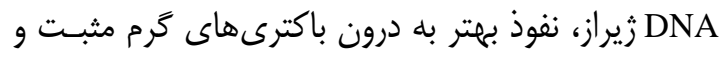

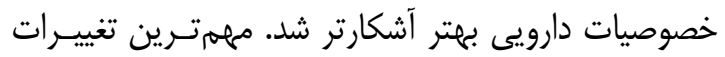

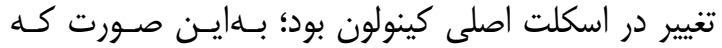

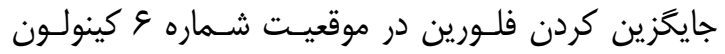

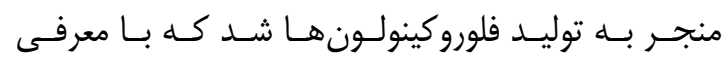
نورفلوكساسين در سال عمل19 و سييروفلوكساسين در سال

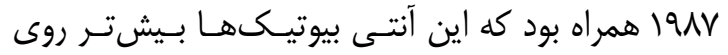

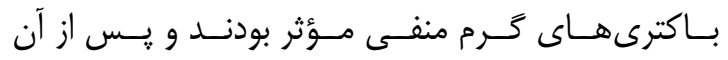

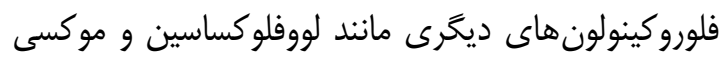

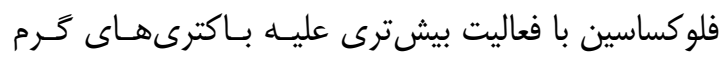
مثبت معرفى شدند.(اوبا) بلددليل قدرت بالا، طيـف فعاليـت كســرده، مصـرف

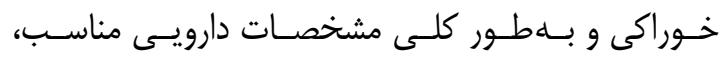

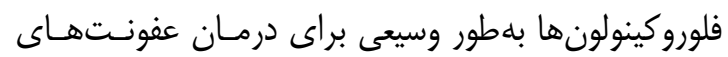

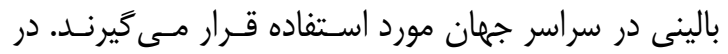

در حال حاضر، يكى از اساسىتـرين مشـكلات قابـل

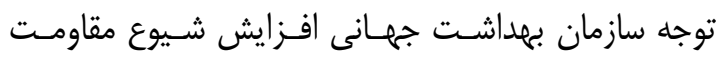

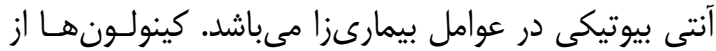

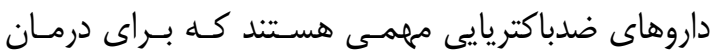

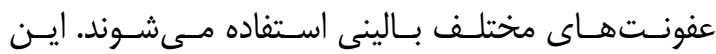

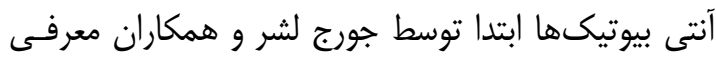
شدند.(') بهعلت استفاده بيش از حلد، ميزان مقاومـتهـاى

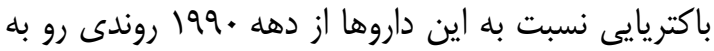

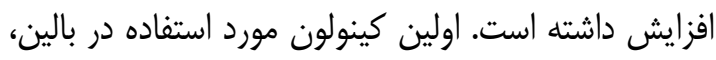

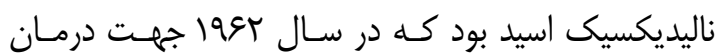
عفونتهاى يبجيـيـده مجـارى ادرارى مـورد اسـتفاده قـرار

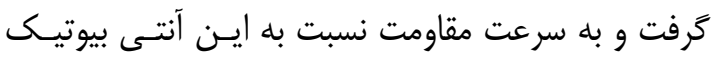

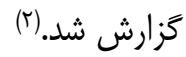

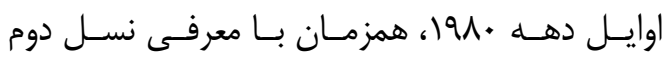
كينولونها، نقش مهم آنها جهت فعاليت مؤثر عليه آنزيم 


\section{طبقهبندى داروهاى كينولون:}

تاكنون كينولونها در قالب جهار نسل معرفى شـدند

كه عبارتند از:

الف) نسل اول شامل: ناليديكسيك اسـيد، سينو كساسـين،

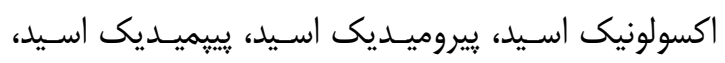
روزوكساسين، فلومكوئين.

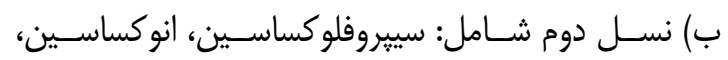

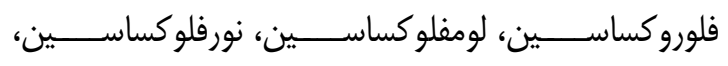

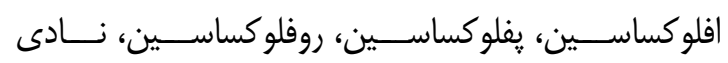
فلوكساسين. ج) نسل سوم شـامل: لووفلو كساسـين، اسيارفلو كساسـين،

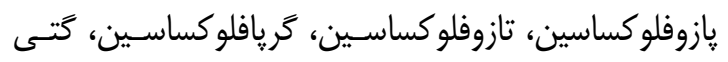
فلوكساسين.

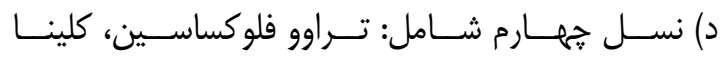

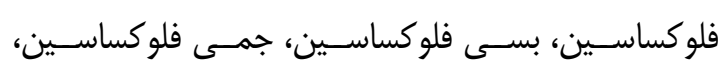

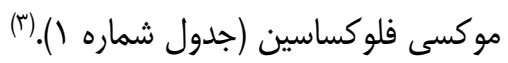

روند توسعه اين داروها، تغييـر هسـته كينولـون از طريـق

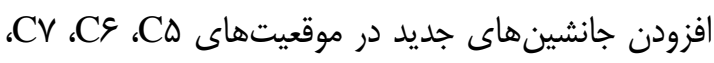

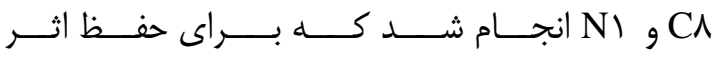

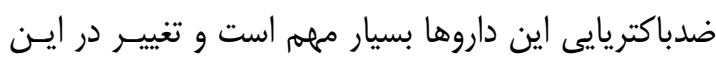

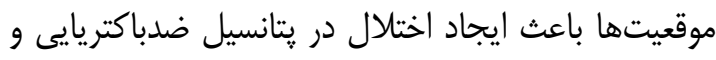

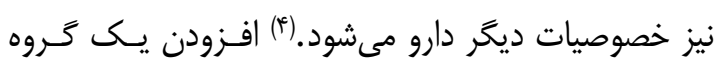

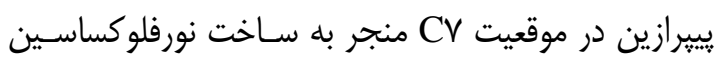

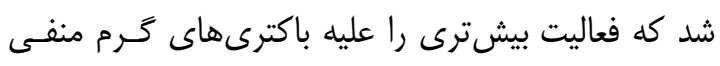

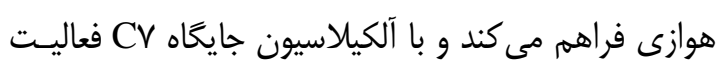

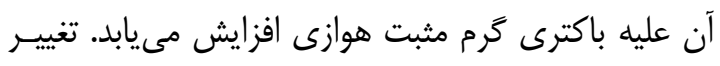

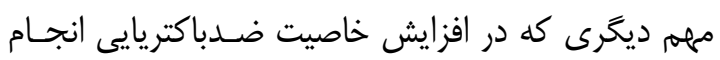
شد، جايخزينى كروه كتون و ب-كربو كسيل در جايكاه

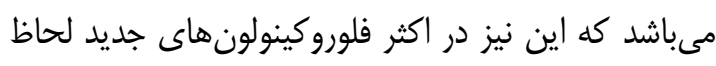

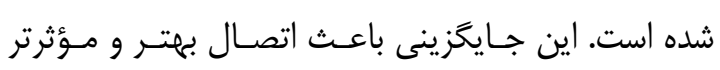

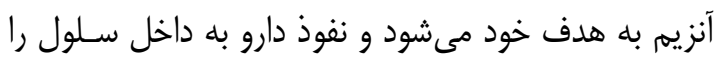
تسهيل مى كند.(ه)

\section{جدول ا - طبقهبندى داروهاى كينولون و طيف عملكرد آنها}

\begin{tabular}{|c|c|c|}
\hline عملكرد & نام داروها & نسل \\
\hline 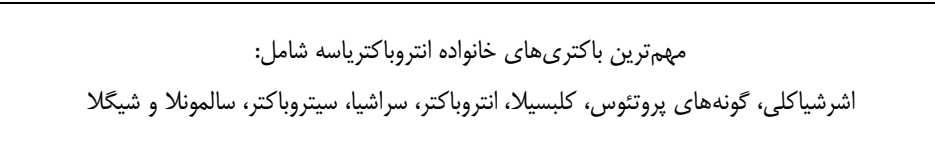 & 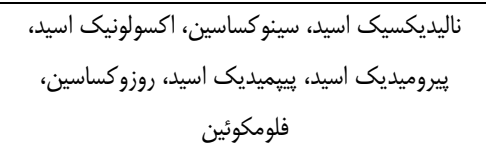 & اول \\
\hline 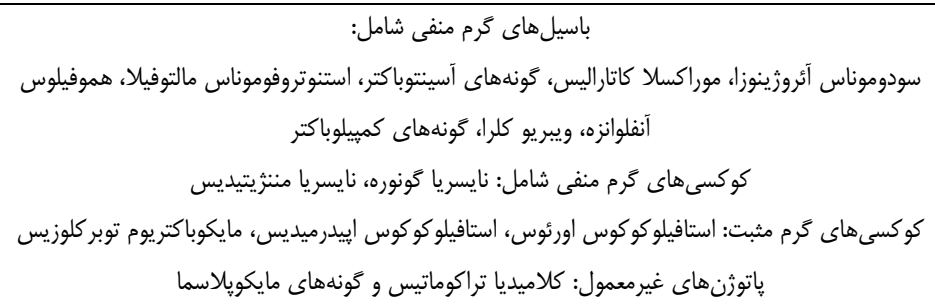 & 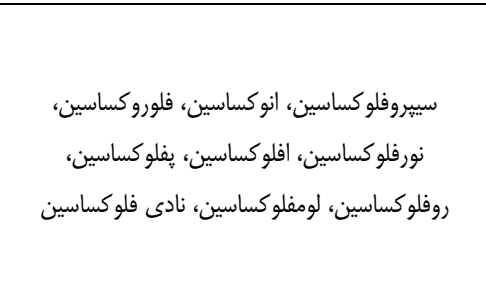 & دوم \\
\hline 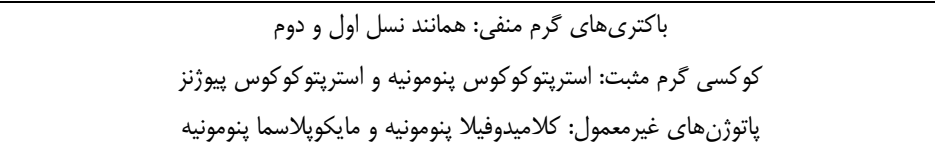 & 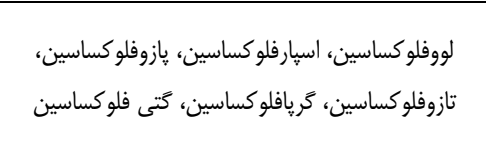 & سوم \\
\hline 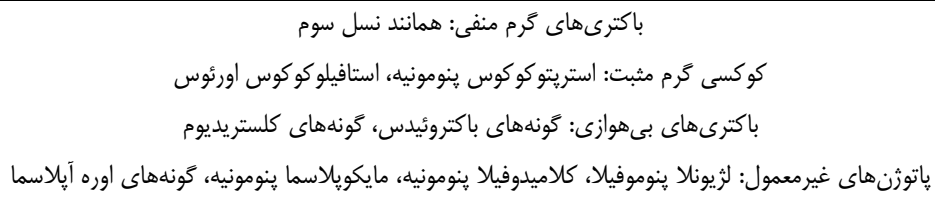 & جمى فلوكساسين، تراوو فلوكساسين، كلينا فلوكساسين، & הهارم \\
\hline
\end{tabular}


آنتى بيوتيك كم شده و حساسيت باكترى نسبت به مـاده

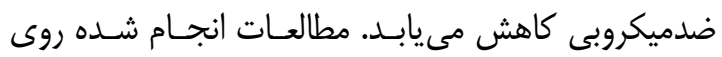

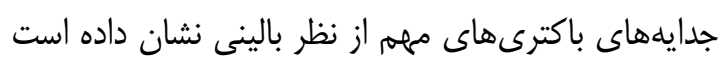

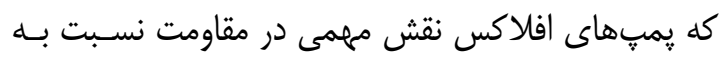

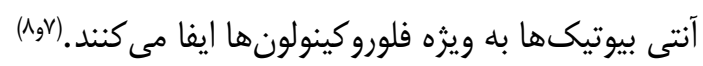

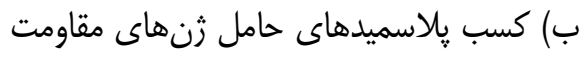

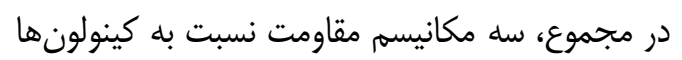
به واسطه پالاسميد وجود دارد كه شامل موارد زير مىباشد:

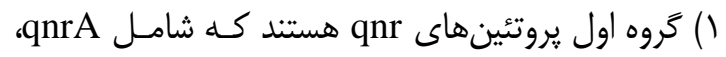

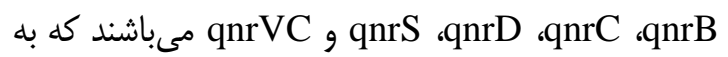
خانواده ينتاييتيدهاى تكرارى تعلق دارند و با مهار اتصـال

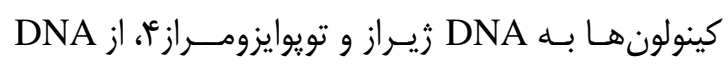

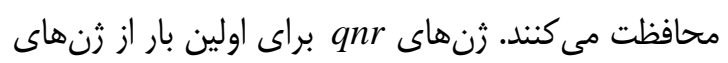

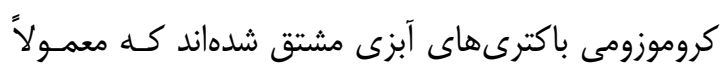

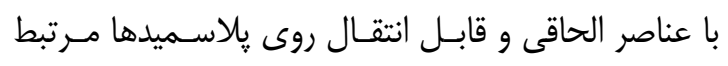

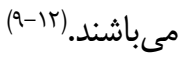

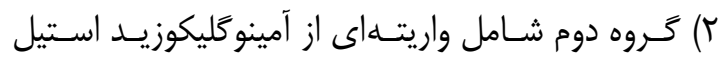

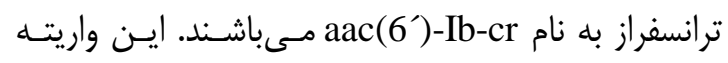

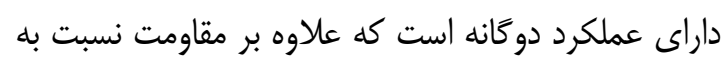

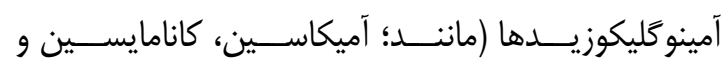
توبرامايسين)، توانايى استيله كردن فلوروكينولونها (مانند

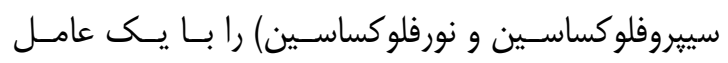

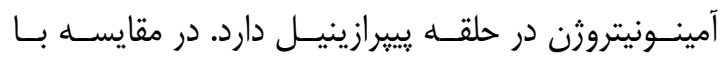

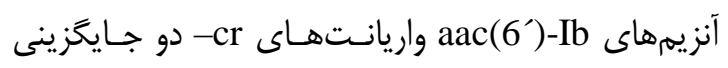

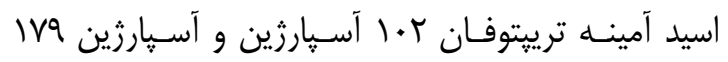

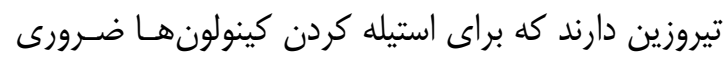

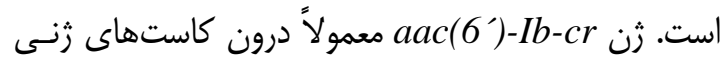

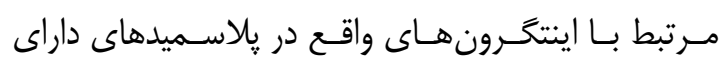
مقاومت جند مَانه يافت مىشوند.

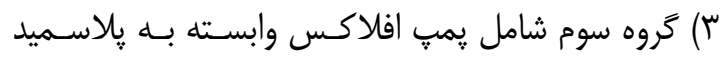

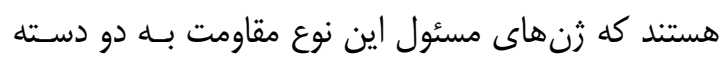
OqxAB و QepAB

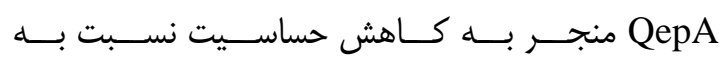

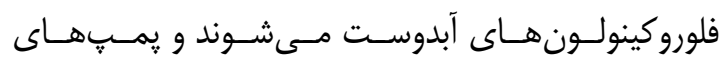

نورفلوكساسين از اولين داروهاى كينولون با طيف اثر

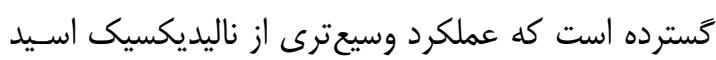
دارد ولى بهعلت سطح سرمى بالا و نفوذ به درون بافتهان اليكا،

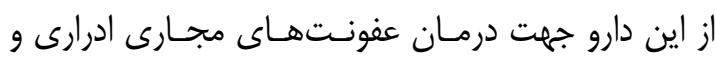

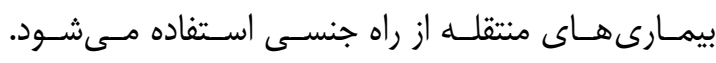

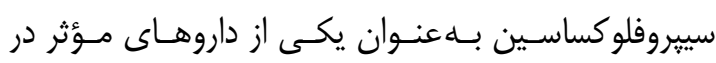

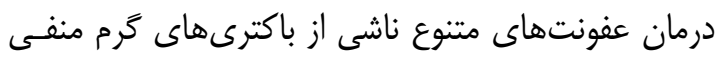

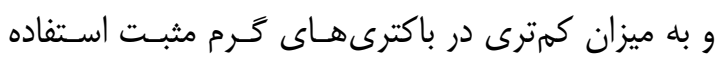

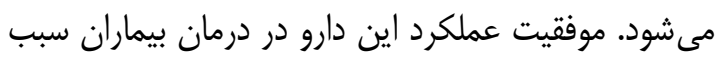

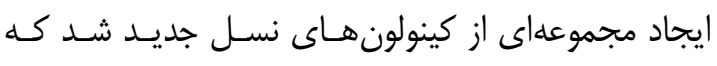

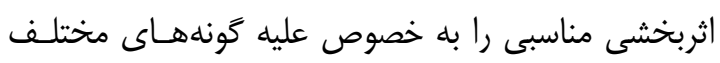

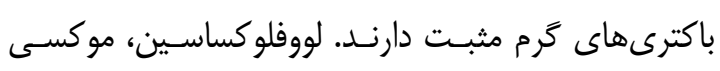

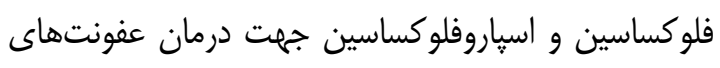
تنفسى ناشى از باكترىهاى كرم مثبت مورد استفاده قرار

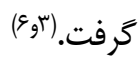
مكانيسمهاى مقاومت نسبت به داروهاى كينولون: با توسعه ييشـرفت داروهـاى ايسن خـانواده همزمـان

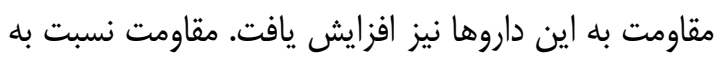
اين داروها به دو مكانيسم عمده انجام مى شود:

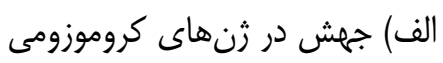

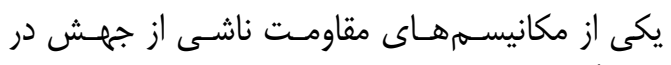

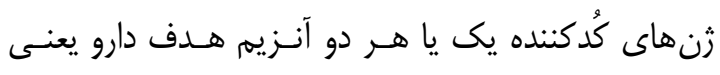

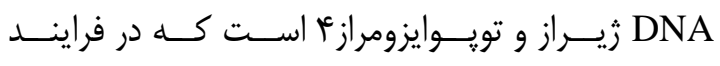

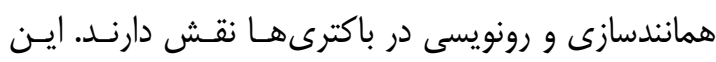
جهش ها بلهترتيب در دومـنهـاى ParC ، gyrB ، gyrA

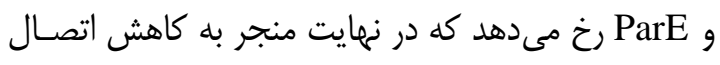

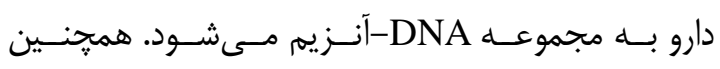
جهش هايى كه باعث كـاهش غلظـت داخـل سـلولى دارو

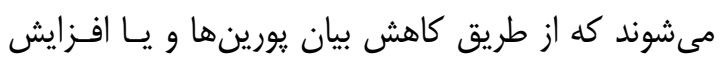

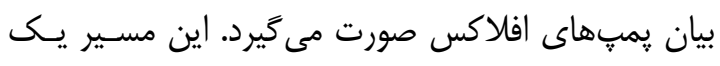

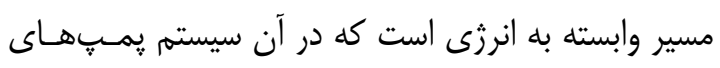

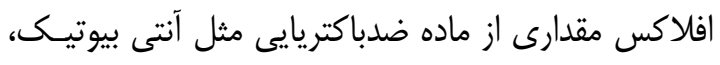

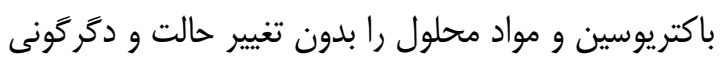

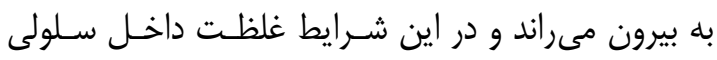




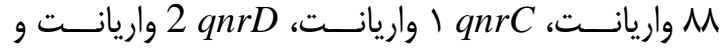

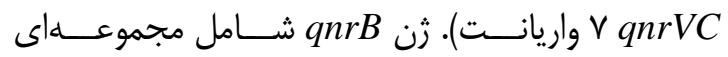

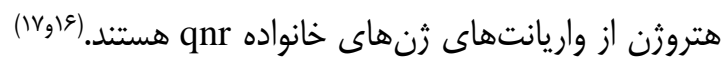

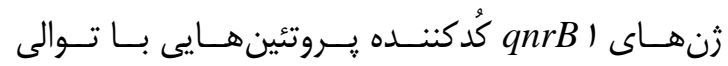

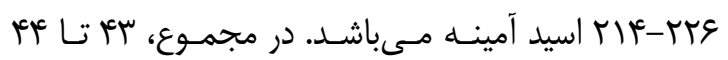

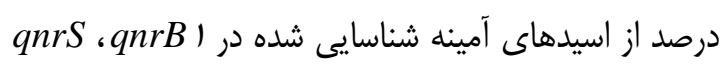

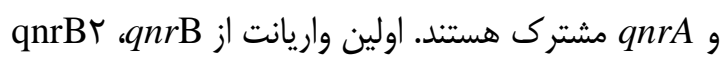

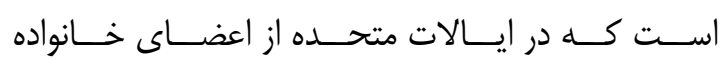

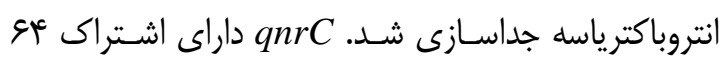

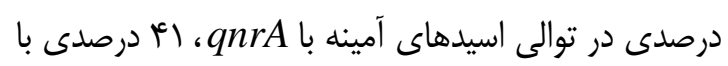

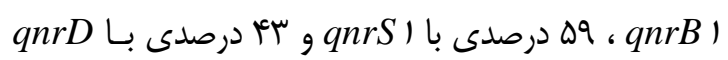

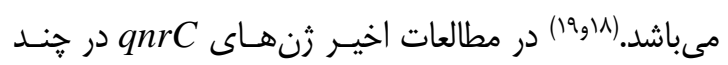

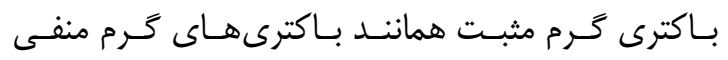

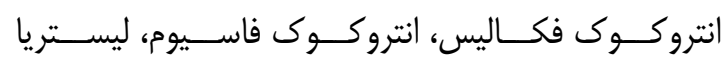

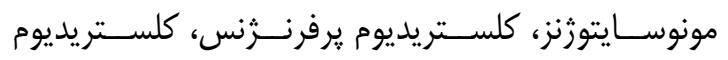

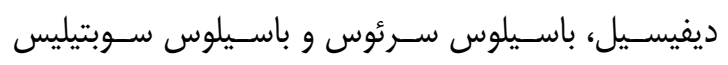

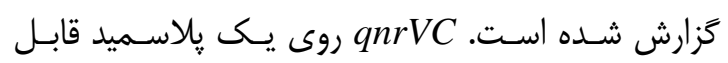

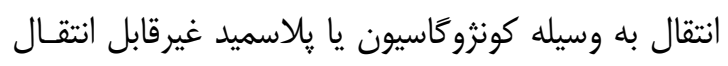

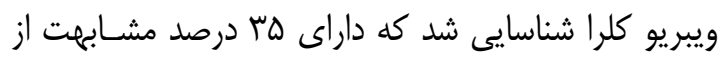

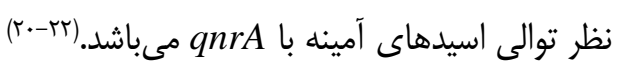

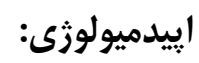

طى دهل كذشته، مقاومت به كينولونهـا بــهـ واسـطه

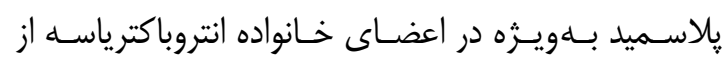
سراسر جهان كزارش مى شود. در ارويا، بريالس و همكاران

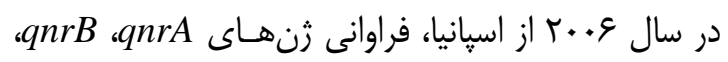

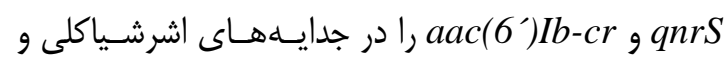

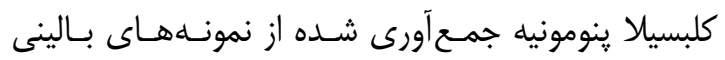

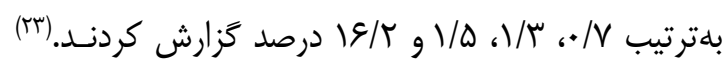

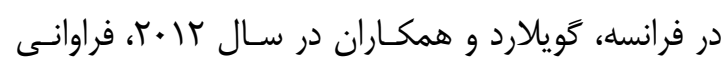

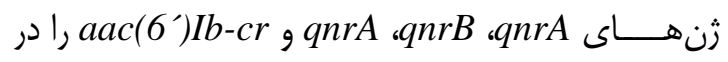

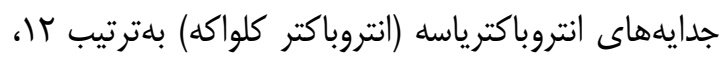

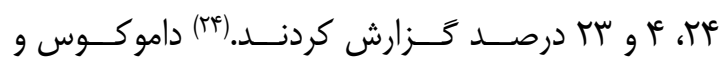

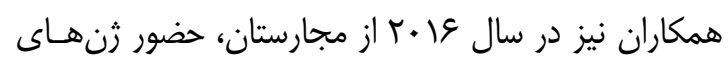

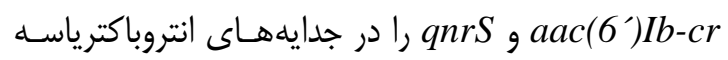

نيز علاوه بر مقاومت نسبت به فلوكينولـونهـا OqxAB

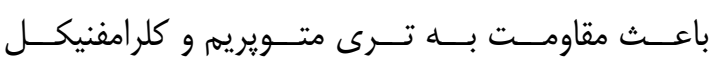
مى شوند. (آوساع)

يروتئين هاى "qur:

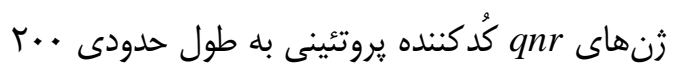

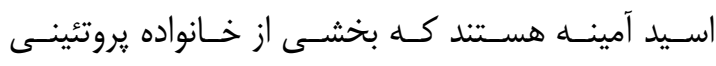

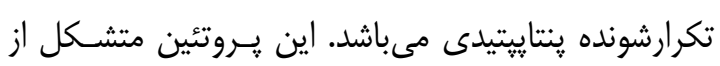

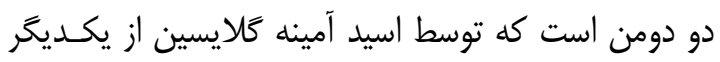
جدا مىشوند و در موقعيت i داراى اسيد آمينـه سيسـتئين

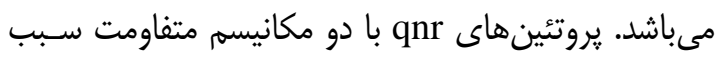
مقاومت نسبت به كينولونها مى شوند:

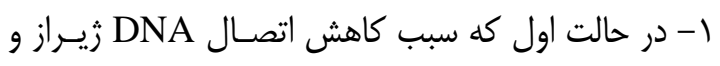

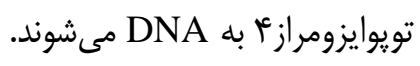

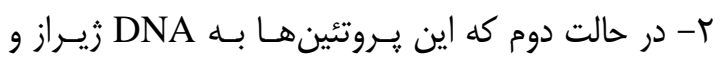

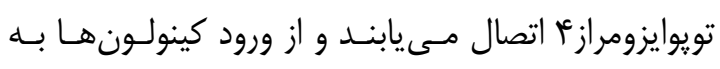
قسمتهاى شكسته شده توسط آنزيم ممانعت مى كنند. به

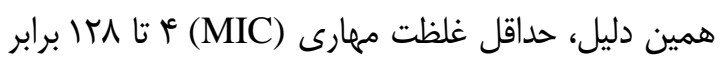

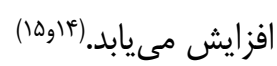

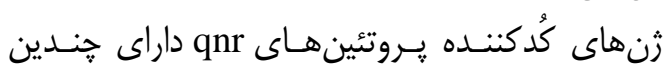

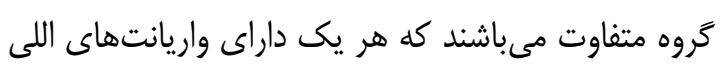

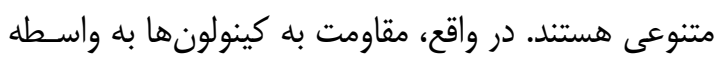

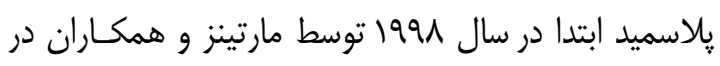
يك جدايه كلبسيلا ينومونيه جدا شده از يكى بيمار بسترى دارئ

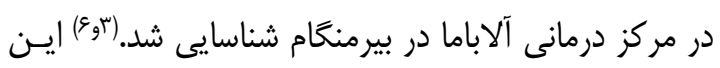

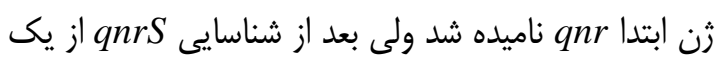

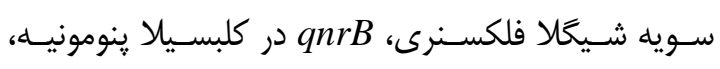

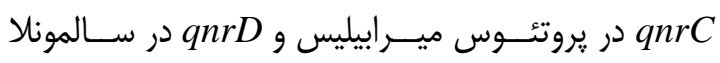

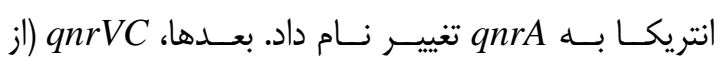

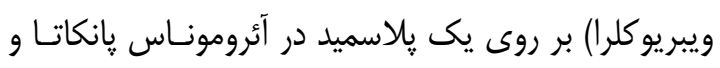

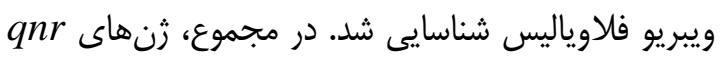

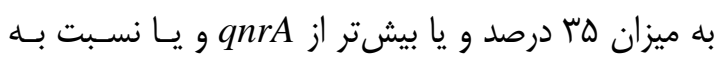
يكديخر تفاوت توالى دارند. علاوه بر اين، اكثر اين زن إنهـا

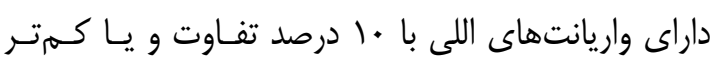

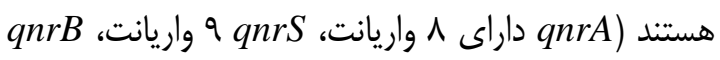


در آسيا و در ايران مطالعات انجام شده حاكى از بـروز

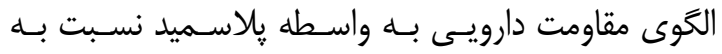

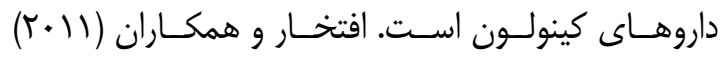

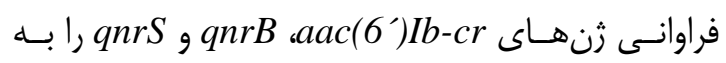

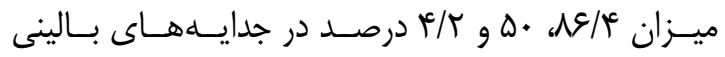

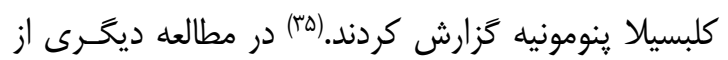

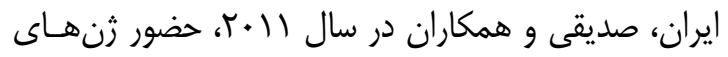

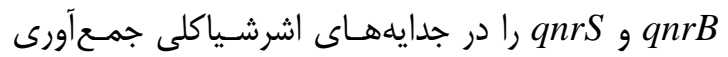

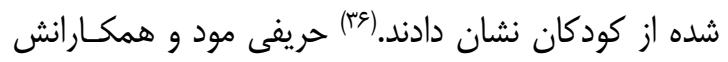
در سال ها •r، فراوانى ثنهاى qurA و

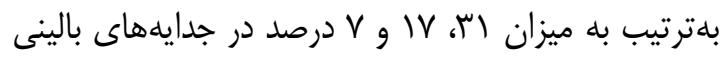

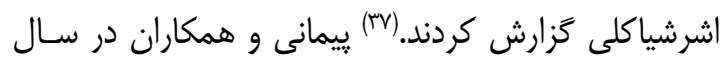

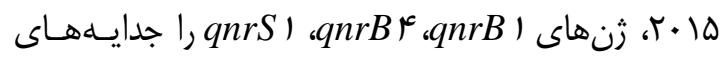

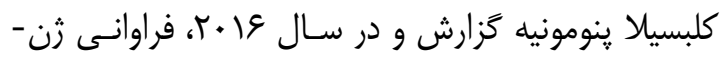

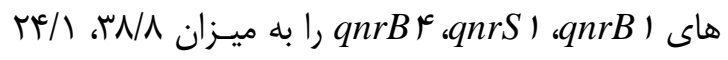

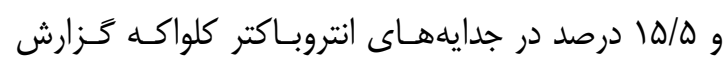

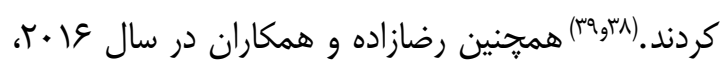

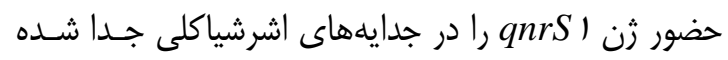

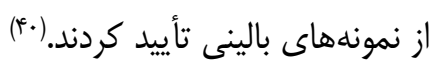

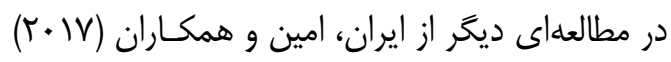

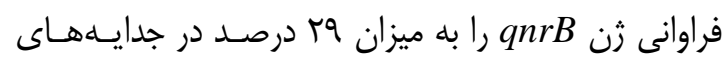

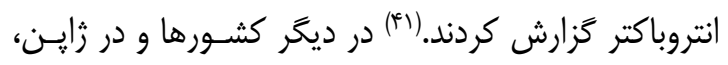

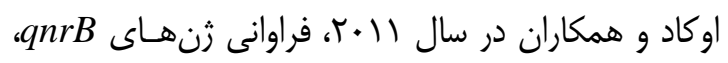

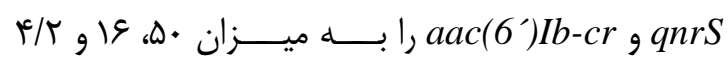

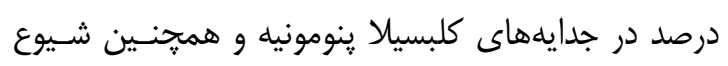

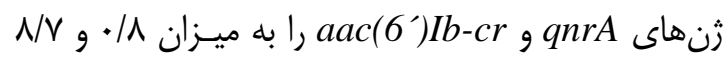

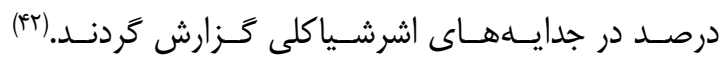

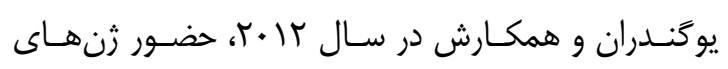

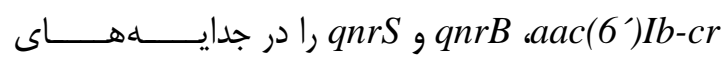

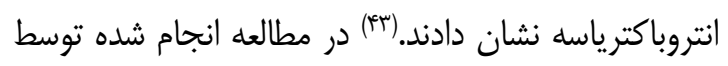

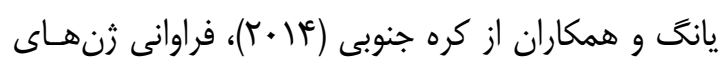

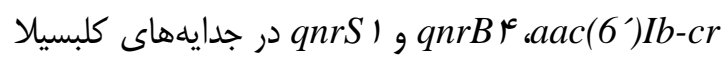

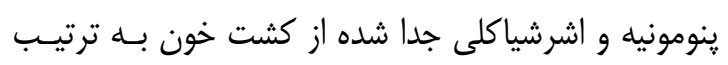

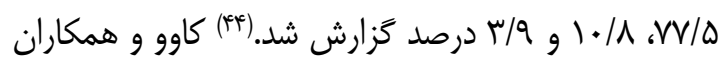

جدا شده از بيماران مبتلا بـهـ عفونـتهــاى خـونى نشـان

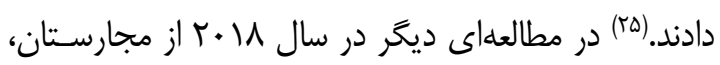

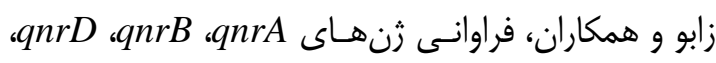

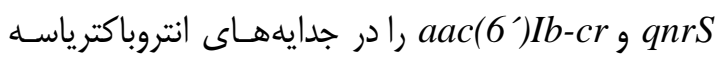

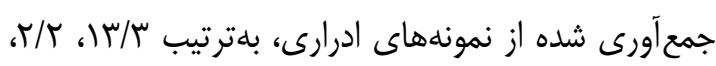

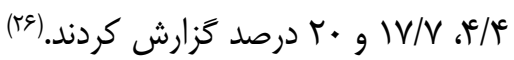

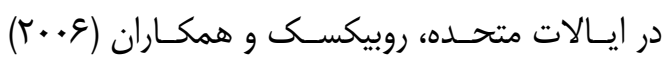

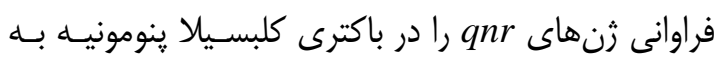

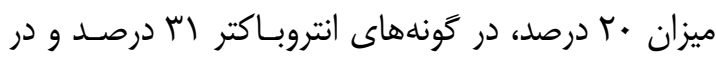

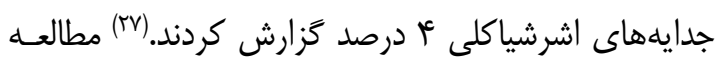

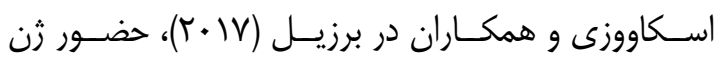

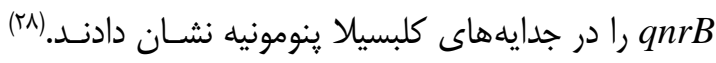

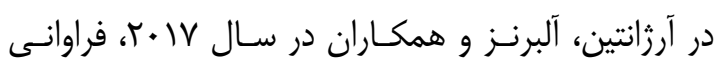

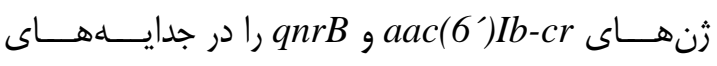

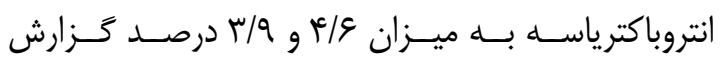

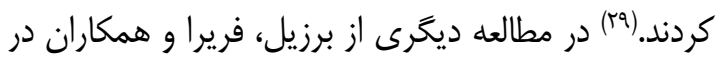

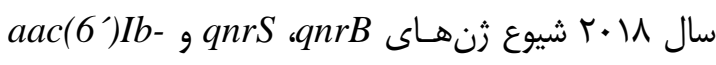

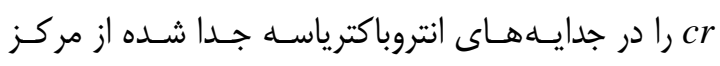

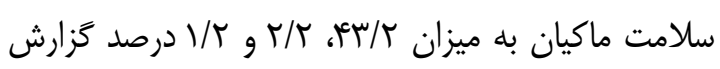
كردند. (ץ) در آفريقا، منيف و همكاران در سـال ||(+r، فراوانسى

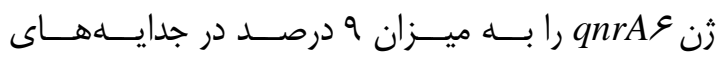

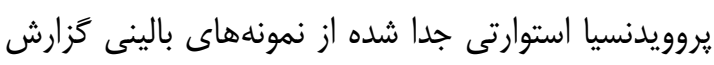

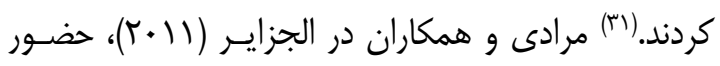

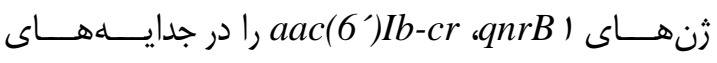

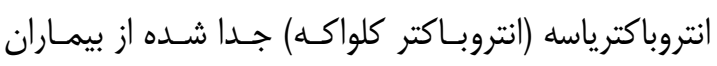

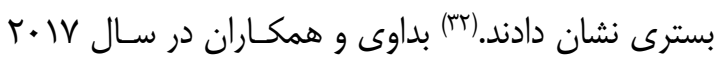

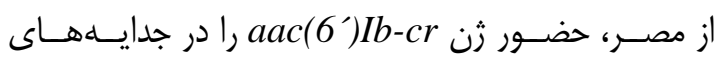

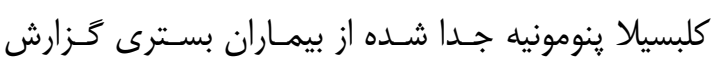

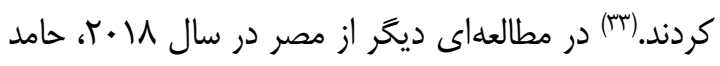

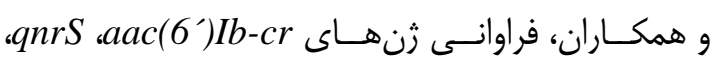

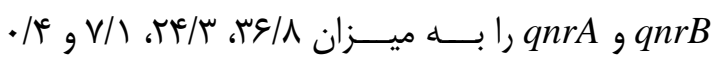

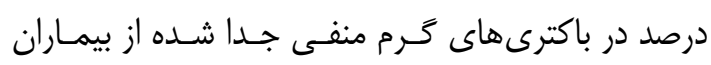
سرطانى گزارش كردند.(r) 
gatifloxacin, levofloxacin, and norfloxacin MICs for fluoroquinolone - resistant Escherichia coli clinical isolates. Antimicrob Agents Chemother 2009; 53(1): 229-34. doi: 10.1128/AAC.00722-08.

5. Hooper DC. Emerging mechanisms of fluoroquinolone resistance. Emerg Infect Dis 2001; 7(2): 337-41. doi: 10.3201/eid0702. 700337.

6. Martínez-Martínez L, Pascual A, García I, Tran J, Jacoby GA. Interaction of plasmid and host quinolone resistance. J Antimicrob Chemother 2003; 51(4): 1037-9. doi: 10. 1093/jac/dkg157.

7. Ghanbari R, Shahryari A, Asgari E, Hosseinpoor S, Yeganeh J, Salighehdar Iran $\mathrm{N}$, et al. Environmental cycle of antibiotic resistance encoded genes: A systematic review. J Qazvin Univ Med Sci 2017; 21(5): 71-55. [In Persian]

8. Jacoby GA, Strahilevitz J, Hooper DC. Plasmid-mediated quinolone resistance. Microbiol Spectr 2014; 2(5). doi: 10.1128/ microbiolspec.PLAS-0006-2013.

9. Cayci YT, Coban AY, Gunaydin M. Investigation of plasmid-mediated quinolone resistance in Pseudomonas aeruginosa clinical isolates. Indian J Med Microbiol 2014; 32(3): 285-9. doi: 10.4103/02550857.136567.

10. Cattoir V, Nordmann P. Plasmidmediated quinolone resistance in gram negative bacterial species: an update. Curr Med Chem 2009; 16(8): 1028-46. doi: 10. 2174/092986709787581879.

11. Soleimani-asl Y, Zibaei M, Firoozeh F. Detection of qnrA gene quinolone-resistant Escherichia coli isolated from urinary tract infections in Khorram Abad during 20112012. Feyz 2013; 17(5): 488-94. [In Persian] 12. Pan XS, Yague G, Fisher LM. Quinolone resistance mutations in Streptococcus
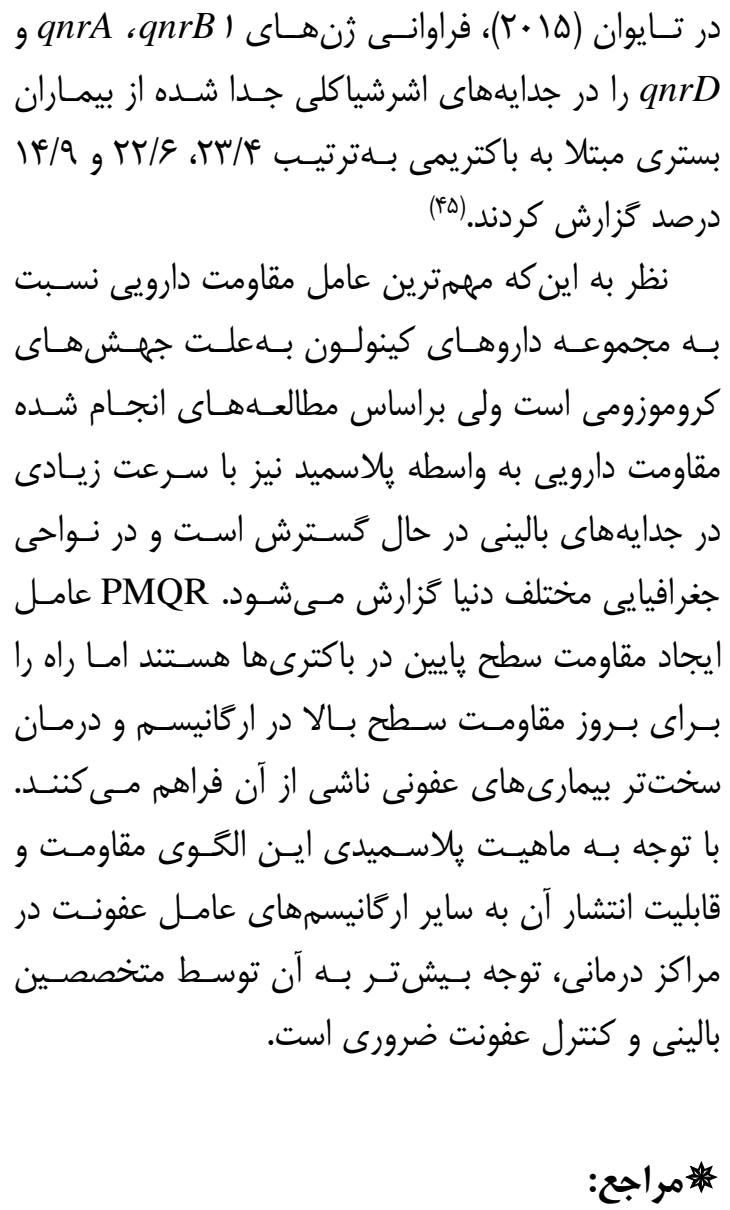

1. Lesher GY, Froelich EJ, Gruett MD, Bailey JH, Brundage RP. 1,8-Naphthyridine derivatives. A new class of chemotherapeutic agents. J Med Chem 1962; 5(5): 1063-5. doi: 10.1021/jmo12409021.

2. Aldred KJ, Kerns RJ, Osheroff $\mathrm{N}$. Mechanism of quinolone action and resistance. Biochemistry 2014; 53(10): 156574. doi: 10.1021/bi5000564.

3. Yanat B, Rodríguez-Martínez JM, Touati A. Plasmid-mediated quinolone resistance in Enterobacteriaceae: a systematic review with a focus on Mediterranean countries. Eur $\mathrm{J}$ Clin Microbiol Infect Dis 2017; 36(3): 42135. doi: 10.1007/s10096-016-2847-x.

4. Becnel Boyd L, Maynard MJ, MorganLinnell SK, Horton LB, Sucgang R, Hamill RJ, et al. Relationships among ciprofloxacin, 
pneumoniae GyrA and ParC proteins: mechanistic insights into quinolone action from enzymatic analysis, intracellular levels, and phenotypes of wild-type and mutant proteins. Antimicrob Agents Chemother 2001; 45(11): 3140-7. doi: 10.1128/AAC. 45.11.31403147.2001.

13. Jiang Y, Zhou Z, Qian Y, Wei Z, Yu Y, $\mathrm{Hu} \mathrm{S}$, et al. Plasmid-mediated quinolone resistance determinants $q n r$ and $a a c\left(6^{\prime}\right)-I b-c r$ in extended-spectrum $\beta$-lactamase-producing Escherichia coli and Klebsiella pneumoniae in China. J Antimicrob Chemother 2008; 61(5): 1003-6. doi: 10.1093/jac/dkn063.

14. Chopra S, Galande A. A fluoroquinoloneresistant Acinetobacter baumannii without the quinolone resistance-determining region mutations. J Antimicrob Chemother 2011; 66(11): 2668-70. doi: 10.1093/jac/dkr364.

15. Vakili B, Khorvash F, Fazeli H, Khaleghi M. Detection of quinolone-resistance mutations of $\operatorname{parC}$ gene in clinical isolates of Acinetobacter baumannii in Iran. J Res Med Sci 2014; 19(6): 567-70.

16. Li Z, Deguchi T, Yasuda M, Kawamura T, Kanematsu E, Nishino Y, et al. Alteration in the GyrA subunit of DNA Gyrase and the ParC subunit of DNA Topoisomerase IV in quinolone-resistant clinical isolates of Staphylococcus epidermidis. Antimicrob Agents Chemother 1998; 42(12): 3293-5.

17. Firoozeh F, Zibaei M, Soleimani-Asl Y. Detection of plasmid-mediated $q n r$ genes among the quinolone-resistant Escherichia coli isolates in Iran. J Infect Dev Ctries 2014; 8(7): 818-22. doi: 10.3855/jidc.3746.

18. Ranjbar R, Behnood V, Memariani H, Najafi A, Moghbeli M, et al. Molecular characterisation of quinolone-resistant Shigella strains isolated in Tehran, Iran. J Glob Antimicrob Resist 2016; 5: 26-30. doi: 10.1016/j.jgar.2016.01.010.

19. Guan X, Xue X, Liu Y, Wang J, Wang Y, Wang $\mathrm{J}$, et al. Plasmid-mediated quinolone resistance current knowledge and future perspectives. J Int Med Res 2013; 41(1): 2030. doi: 10.1177/0300060513475965.

20. Jeong HS, Bae IK, Shin JH, Jung HJ, Kim $\mathrm{SH}$, Lee JY, et al. Prevalence of plasmidmediated quinolone resistance and its association with extended-spectrum betalactamase and AmpC beta-lactamase in Enterobacteriaceae. Korean J Lab Med 2011; 31(4): 257-64. doi: 10.3343/kjlm.2011.31.4. 257.

21. Hassan WM, Hashim A, Domany R. Plasmid mediated quinolone resistance determinants $q n r$, aac(6)-Ib-cr, and qep in ESBL producing Escherichia coli clinical isolated from Egypt. Indian J Med Microbiol 2012; 30(4): 442-7. doi: 10.4103/0255-0857. 103766.

22. Fabrega A, Madurga S, Giralt E, Vila J. Mechanism of action of and resistance to quinolones. Microb Biotechnol 2009; 2(1): 40-61. doi: 10.1111/j.17517915.2008.00063. $\mathrm{x}$.

23. Briales A, Rodríguez-Martínez JM, Velasco C, de Alba PD, Rodríguez-Baño J, Martínez-Martínez L, et al. Prevalence of plasmid-mediated quinolone resistance determinants $\quad q n r$ and $a a c\left(6^{\prime}\right)-I b-c r \quad$ in Escherichia coli and Klebsiella pneumoniae producing extended-spectrum $\beta$-lactamases in Spain. Int J Antimicrob Agents 2012; 39(5): 431-4. doi:10.1016/j.ijantimicag.2011.12.009. 24. Guillard T, Cholley P, Limelette A, Hocquet D, Matton L, Guyeux C, et al. Fluoroquinolone resistance mechanisms and population structure of Enterobacter cloacae non-susceptible to ertapenem in NorthEastern France. Front Microbiol 2015; 6: 
1186. doi: 10.3389/fmicb.2015.01186.

25. Domokos J, Kristóf K, Szabó D. Plasmidmediated quinolone resistance among extended spectrum beta lactase producing Enterobacteriaceae from bloodstream infections. Acta Microbiol Immunol Hung 2016; 63(3): 313-23. doi: 10.1556/030. 63.2016.002.

26. Szabó O, Gulyás D, Szabó N, Kristóf K, Kocsis B, Szabó D. Plasmid-mediated quinolone resistance determinants in Enterobacteriaceae from urine clinical samples. Acta Microbiol Immunol Hung 2018; 23: 1-11. doi: 10.1556/030.652018.012. 27. Robicsek A, Strahilevitz J, Sahm DF, Jacoby GA, Hooper DC. Qnr prevalence in ceftazidime-resistant Enterobacteriaceae isolates from the United States. Antimicrob Agents Chemother 2006; 50(8): 2872-74. doi: 10.1128/AAC.01647-05.

28. Scavuzzi AML, Maciel MAV, de Melo HRL, Alves LC, Brayner FA, Lopes ACS. Occurrence of qnrBl and qnrB12 genes, mutation in gyrA and ramR, and expression of efflux pumps in isolates of Klebsiella pneumoniae carriers of blaKPC-2. J Med Microbiol 2017; 66(4): 477-84. doi: 10.1099/ jmm.0.000452.

29. Albornoz E, Lucero C, Romero G, Quiroga MP, Rapoport M, Guerriero L, et al. Prevalence of plasmid-mediated quinolone resistance genes in clinical Enterobacteria from Argentina. Microb Drug Resist 2017; 23(2): 177-87. doi: 10.1089/mdr.2016.0033. 30. Ferreira JC, Penha Filho RAC, Kuaye APY, Andrade LN, Berchieri Junior A, Darini ALDC. Identification and characterization of plasmid-mediated quinolone resistance determinants in Enterobacteriaceae isolated from healthy poultry in Brazil. Infect Genet Evol 2018; 60:
66-70. doi: 10.1016/j.meegid.2018.02.003.

31. Mnif B, Ktari S, Chaari A, Medhioub F, Rhimi F, Bouaziz M, et al. Nosocomial dissemination of Providencia stuartii isolates carrying bla OXA -48, bla $a_{\mathrm{PER}-1}, \quad b l a_{\mathrm{CMY}-4}$ and qnrA6 in a Tunisian hospital. J Antimicrob Chemother 2013; 68(2): 329-32. doi: 10. 1093/jac/dks386.

32. Meradi L, Djahoudi A, Abdi A, Bouchakour M, Perrier Gros Claude JD, Timinouni M. Qnr and aac(6')-Ib-cr types quinolone resistance among Enterobacteriaceae isolated in Annaba, Algeria. Pathol Biol (Paris) 2011; 59(4): e738. doi: 10.1016/j.patbio.2009.05.003.

33. El-Badawy MF, Tawakol WM, El-Far SW, Maghrabi IA, Al-Ghamdi SA, Mansy MS, et al. Molecular identification of aminoglycoside-modifying enzymes and plasmid-mediated quinolone resistance genes among Klebsiella pneumoniae clinical isolates recovered from Egyptian Patients. Int J Microbiol 2017; 2017: 8050432. doi: 10. 1155/2017/8050432.

34. Hamed SM, Aboshanab KMA, ElMahallawy HA, Helmy MM, Ashour MS, Elkhatib WF. Plasmid-mediated quinolone resistance in gramnegative pathogens isolated from cancer patients in Egypt. Microb Drug Resist 2018; 13. doi: 10.1089/mdr.2017.0354. 35. Eftekhar F, and Seyedpour SM. Prevalence of $q n r$ and aac(6')-Ib-cr genes in clinical isolates of Klebsiella Pneumoniae from Imam Hussein hospital in Tehran. Iran J Med Sci 2015; 40(6): 515-21.

36. Sedighi I, Arabestani MR, Rahimbakhsh A, Karimitabar Z, Alikhani MY. Dissemination of extended-spectrum $\beta$ lactamases and quinolone resistance genes among clinical isolates of uropathogenic Escherichia coli in Children. Jundishapur 
J Microbiol. 2015; 8(7): e19184.

37. Harifi Mood E, Meshkat Z, Izadi N, Rezaei M, Amel Jamehdar S, Naderi Nasab M. Prevalence of quinolone resistance genes among extended-spectrum $\beta$-lactamaseproducing Escherichia coli in Mashhad, Iran. Jundishapur J Microbiol 2015; 8(12): e16217. doi: 10.5812/jjm.16217.

38. Peymani A, Naserpour Farivar T, Nikooei L, Najafipour R, Javadi A, Pahlevan AA. Emergence of plasmid-mediated quinoloneresistant determinants in Klebsiella pneumoniae isolates from Tehran and Qazvin provinces, Iran. J Prev Med Hyg 2015; 56(2): E61-5.

39. Peymani A, Farivar TN, Najafipour R, Mansouri S. High prevalence of plasmidmediated quinolone resistance determinants in Enterobacter cloacae isolated from hospitals of the Qazvin, Alborz, and Tehran provinces, Iran. Rev Soc Bras Med Trop 2016; 49(3): 286-91. doi: 10.1590/003786820454-2015.

40. Rezazadeh M, Baghchesaraei H, Peymani A. Plasmid-mediated quinolone-resistance (qnr) genes in clinical isolates of Escherichia coli collected from several hospitals of Qazvin and Zanjan provinces, Iran. Osong Public Health Res Perspect 2016; 7(5): 30712. doi: 10.1016/j.phrp.2016.08.003.
41. Amin M, Dibachi S, Shahin M. Prevalence of class 1 integrons and plasmidmediated qnr-genes among Enterobacter isolates obtained from hospitalized patients in Ahvaz, Iran. Infez Med 2017; 25(4): 351-7.

42. Okade H, Nakagawa S, Sakagami T, Hisada H, Nomura N, Mitsuyama J. Characterization of plasmid-mediated quinolone resistance determinants in Klebsiella pneumoniae and Escherichia coli from Tokai, Japan. J Infect Chemother 2014; 20(12): 778-83. doi: 10.1016/j.jiac.2014.08. 018.

43. Yugendran T, Harish BN. High incidence of plasmid-mediated quinolone resistance genes among ciprofloxacin-resistant clinical isolates of Enterobacteriaceae at a tertiary care hospital in Puducherry, India. Peer J 2016; 4: e1995. doi: 10.7717/peerj.1995.

44. Yang HY, Nam YS, Lee HJ. Prevalence of plasmid-mediated quinolone resistance genes among ciprofloxacin-nonsusceptible Escherichia coli and Klebsiella pneumoniae isolated from blood cultures in Korea. Can J Infect Dis Med Microbiol 2014; 25(3): 163-9. 45. Kao $\mathrm{CY}, \mathrm{Wu} \mathrm{HM}$, Lin WH, Tseng $\mathrm{CC}$, Yan JJ, Wang MC, et al. Plasmidmediated quinolone resistance determinants in quinolone-resistant Escherichia coli isolated from patients with bacteremia in a university hospital in Taiwan, 2001-2015. Sci Rep 2016; 6: 32281. doi: 10.1038/srep32281. 